\title{
The effect of pair bonding in Cabrera vole's scent marking
}

\author{
Luis Alexandre Piteira Gomes • \\ Pedro Miguel Pedreirinho Salgado • \\ Eduardo Nuno Barata • António Paulo Pereira Mira
}

Received: 22 January 2013 /Revised: 29 April 2013 / Accepted: 8 May 2013 /Published online: 22 May 2013

(C) Springer-Verlag Berlin Heidelberg and ISPA 2013

\begin{abstract}
The Cabrera vole (Microtus cabrerae) is a rare rodent living in patchy grassy areas of the Iberian Peninsula where unpaired individuals of both sexes use scent marking primarily to increase their mate-finding likelihood. Cabrera voles establish long-term pair bonds with opposite-sex conspecifics constituting a breeding pair, which is expected to reduce the efforts in searching for a new mate. Under such circumstances, scent marking as a strategy to increase matefinding likelihood became useless. Accordingly, we hypothesise that pair bonded Cabrera voles suppress matefinding scent marking to reduce energetic costs and predation risk. To test this hypothesis, we compared scent-marking behaviour towards a clean substrate, in both paired and nonpaired voles. No differences were found in the scent marks' type and the amount of marks placed by voles in both conditions. We also analysed the scent-marking behaviour of both sex pair bonded voles when exposed simultaneously to a clean substrate, a substrate pre-marked by males and a substrate premarked by females. We found no significant differences in scent-marks (urine-marked area and number of faecal boli)
\end{abstract}

L. A. P. Gomes $(\bowtie)$

Grupo de Ecossistemas e Paisagens Mediterrânicas-Instituto de Ciências Agrárias e Ambientais Mediterrânicas, Universidade de Évora-Núcleo da Mitra, Apartado 94,

7002-554 Évora, Portugal

e-mail: luispgomes@gmail.com

L. A. P. Gomes • P. M. P. Salgado • A. P. P. Mira

Unidade de Biologia da Conservação, Universidade de

Evora-Núcleo da Mitra, Apartado 94,

7002-554 Évora, Portugal

E. N. Barata

Departamento de Biologia, Universidade de Évora, Apartado 94, 7002-554 Évora, Portugal

A. P. P. Mira

CIBIO - Centro de Investigação em Biodiversidade e Recursos Genéticos-Pólo de Évora, Évora, Portugal across the three types of substrate types. In accordance with our prediction, these results suggest that pair bonded Cabrera voles did not use scent marking for mate finding, thus providing further support to the existence of a monogamous mating strategy. Furthermore, our results fail to support the use of scent marking for territorial defence purposes.

Keywords Behaviour plasticity $\cdot$ Microtus cabrerae . Modulation $\cdot$ Pair bonding $\cdot$ Scent marking $\cdot$ Voles

\section{Introduction}

The placement of semiochemicals on the substrate to communicate with conspecifics, defined as scent marking, is a common trait among mammals (Johnson 1973). Scent marking typically entails faeces deposition, urine and/or dragging a particular region of the body with scent-producing glands (e.g. the anogenital region) on the substrate (Eisenberg and Kleiman 1972; Ferkin 2001; Ferkin and Johnston 1995), and seems to serve multiple and, occasionally, overlapping functions (Becker et al. 2012; Ferkin et al. 2001, 2004). Scent marking is used mainly for territorial defence, as well as mate advertisement and self-advertisement (Johnston 1983; Thomas and Wolff 2002). Given the high costs of scent marking for both scent donors and receivers (Gosling et al. 2000; Hughes et al. 2010; Koivula and Korpimaki 2001; Mason et al. 2005), it is not surprising that animals may modulate their scent-marking behaviour, reducing costs when scent marking is not profitable (Roberts et al. 2001). Notwithstanding, the plasticity of mammals' scent-marking behaviour at the species level is poorly studied. Only a few studies have focused on the intra-specific plasticity of scent-marking behaviour; these studies show that scent-marking behaviour may respond powerfully to changed social conditions (Becker et al. 2012; Le Roux et al. 2008). For instance, in yellow mongooses (Cynictis penicillata) the population density seems to affect the subordinates' intensity of 